\title{
Erratum
}

\section{Erratum to "Empirical Risk Analysis of Severe Reactor Accidents in Nuclear Power Plants after Fukushima"}

\author{
Jan Christian Kaiser \\ Softwareentwicklung \& Umwelt-Consulting, Kienestraße 1a, 80933 München, Germany \\ Correspondence should be addressed to Jan Christian Kaiser; jan-christian.kaiser@gmx.de \\ Received 15 October 2013; Accepted 4 November 2013; Published 23 January 2014 \\ Copyright (C) 2014 Jan Christian Kaiser. This is an open access article distributed under the Creative Commons Attribution License, \\ which permits unrestricted use, distribution, and reproduction in any medium, provided the original work is properly cited.
}

In the paper an error occurred in the calculation of the cumulative reactor years from the data of IAEA report RDS2/31 (2011 edition). It affects Figure 1 and Table 2 of the original paper. Minor implications in the following sections: Materials and Methods, Results, and Discussion, have been corrected below. The main results of the paper are derived with the correct value of $C_{W}(2010)=14097$ cumulative reactor years for the Fukushima accident. In the Acknowledgment section there, is also a change.

Materials and Methods. The curve of cumulative reactor years is corrected in the new Figure 1. Likewise, values for cumulative reactor years and the corresponding hazards of the TMI accident and of the Chornobyl accident in original Table 2 have to be replaced with those of the new Table 2.

Results. The first two sentences refer to the original Table 2 and are discarded. The last three sentences are replaced by "The empirical estimates for the reactor-specific hazard $h_{\mathrm{emp}}$ decrease from $1.2 \times 10^{-5} \mathrm{RY}^{-1}$ (1979) and $3.3 \times 10^{-6} \mathrm{RY}^{-1}$ (1986) to $4.8 \times 10^{-7} \mathrm{RY}^{-1}$ (2011). The value for 2011 is considered the most reliable because it is derived with the largest numbers of accidents and cumulative reactor years. It falls below the point estimate of $h_{\mathrm{PSA}}=2.5 \times 10^{-6} \mathrm{RY}^{-1}$ from the PSA study [10] and stays within its $95 \%$ CI. Since the PSA considered accidents of INES level $\geq 4$ and $h_{\text {emp }}$ pertains to level $\geq 5$, the results are compatible."
Discussion. In the fourth sentence, the phrase "a factor of three" is replaced by "a factor of 25 ." 
TABLE 2: Worldwide number $R_{W}$ of reactors at the time of accident, cumulative reactor years since 1967, and hazard of NPP reactor accidents with INES level $\geq 5$ (95\% CI in brackets are only calculated for the latest value based on three events).

\begin{tabular}{lccc}
\hline Reactor location & Number of reactors $R_{W}$ & Cumulative reactor years $C_{W}$ since 1967 & Hazard $h_{W}\left(\mathrm{per} 10^{4} \mathrm{RY}\right)$ \\
\hline Three Mile Island & 133 & 631 & 16 \\
Chornobyl & 302 & 2019 & 9.9 \\
Fukushima & 441 & 14097 & 2.1 \\
& & & $(0.44 ; 6.2)$ \\
\hline
\end{tabular}

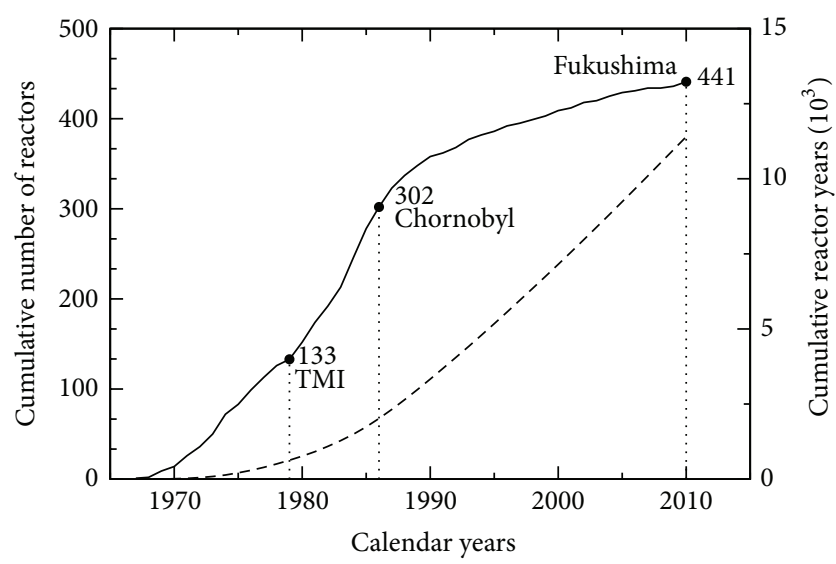

- Cumulative number of reactors

- - Cumulative reactor years

FIGURE 1: Cumulative number and cumulative years of experience for reactors connected to the grid until the end of 2010; reactor numbers in the years 1979, 1986, and 2011 with accidents of INES levels $\geq 5$ are annotated.

\section{Acknowledgment}

The author would like to thank Dr. Yang Duanjie, Beijing, China, for carefully reading the original paper and for reporting the error. 


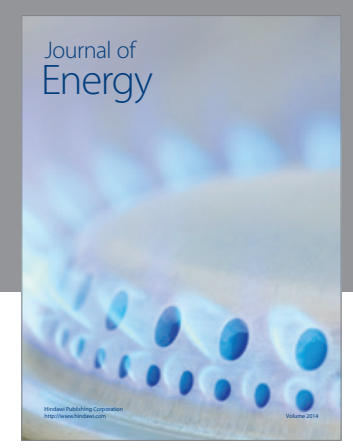

Journal of

Industrial Engineering
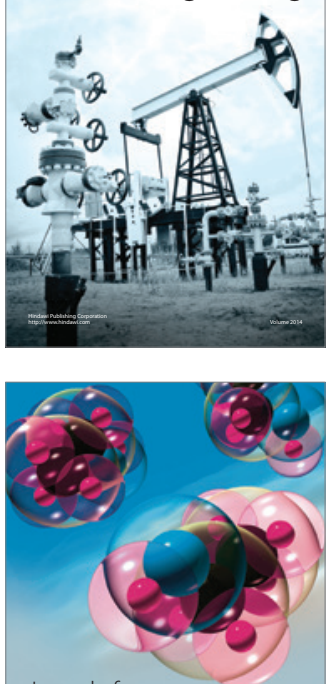

Fuels
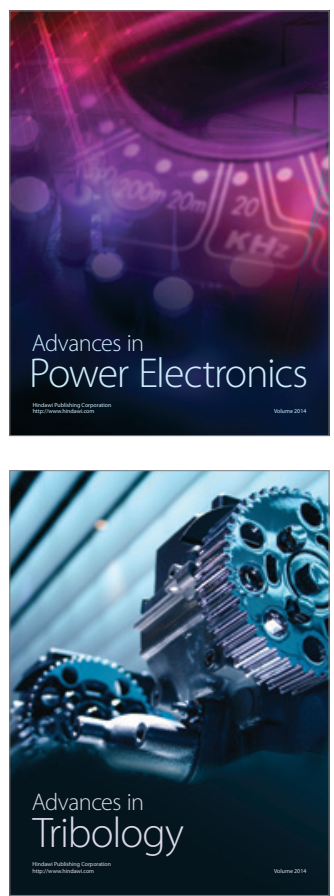

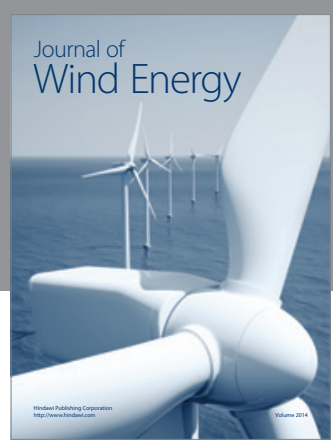

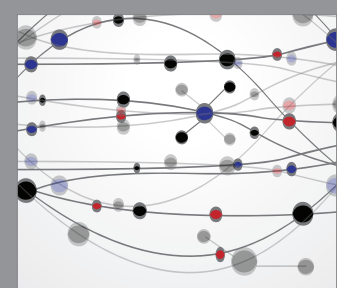

The Scientific World Journal

Submit your manuscripts at http://www.hindawi.com

Journal of

Structures
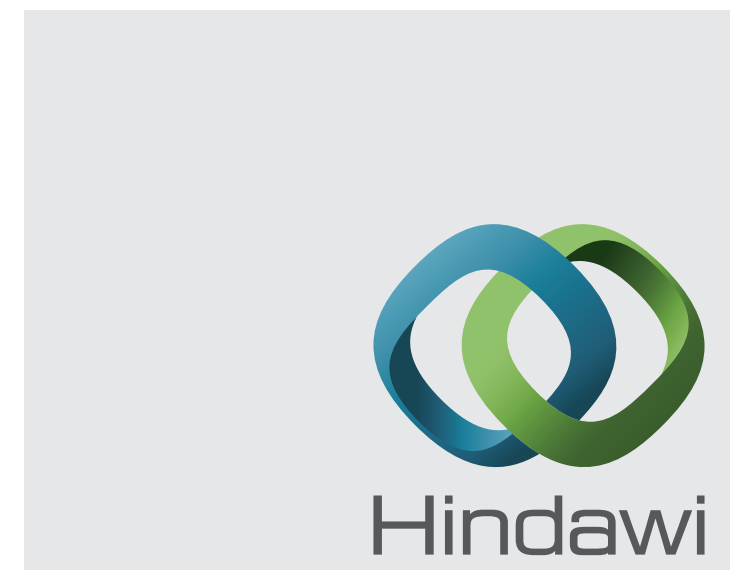

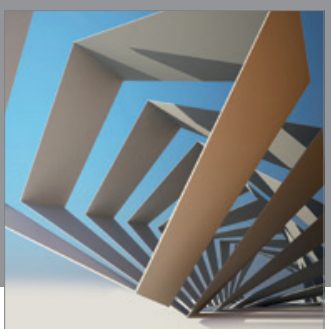

Rotating

Machinery
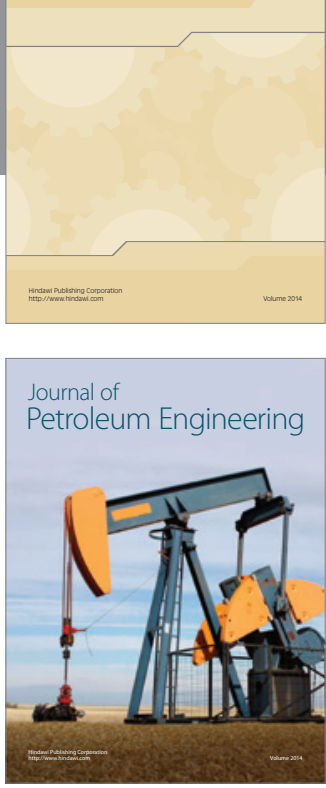

Journal of

Solar Energy
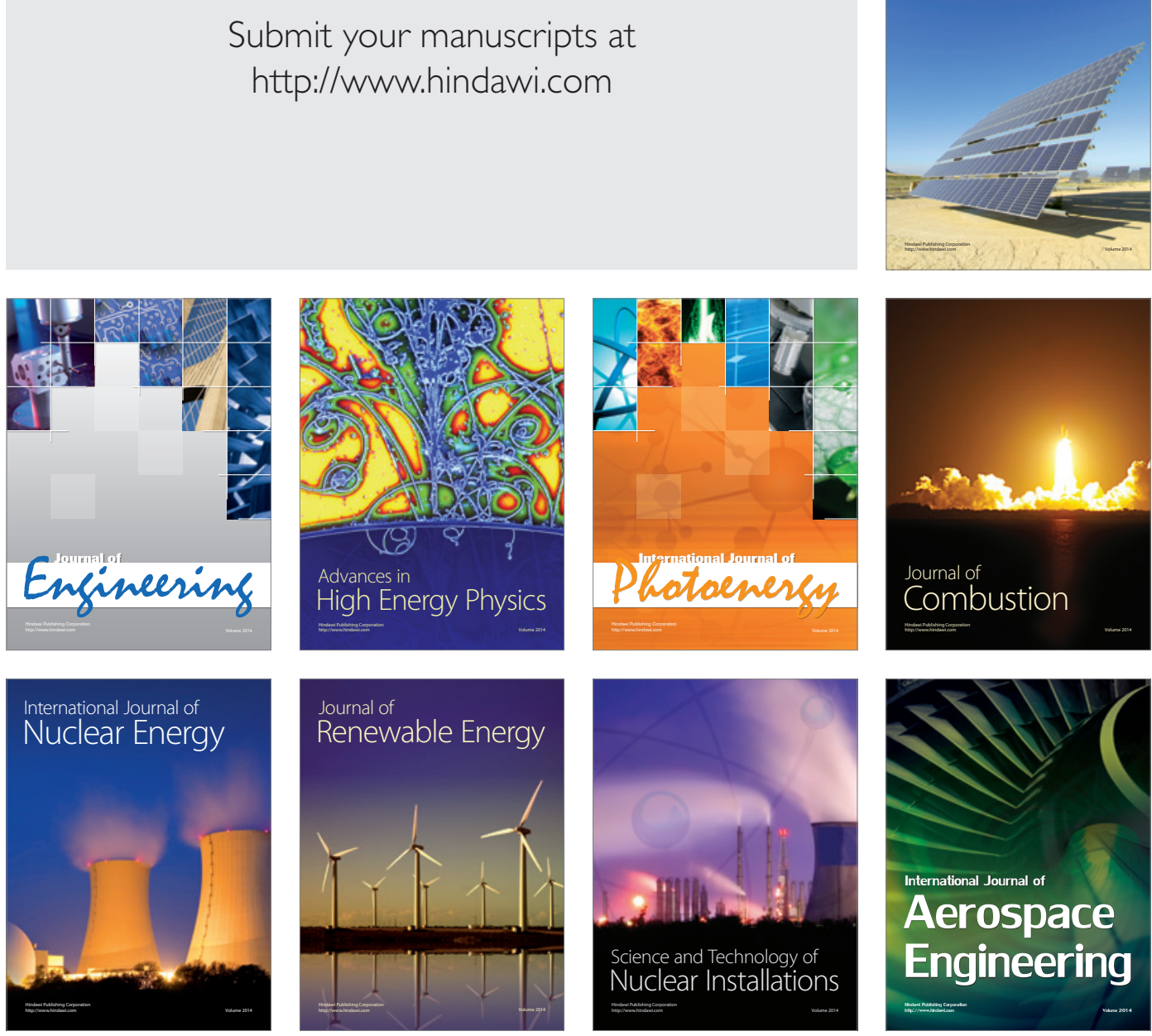